\title{
CARDIAC SARCOIDOSIS- A RARE CASE REPORT IN AUTOPSY SPECIMEN WITH REVIEW OF LITERATURE
}

Shaista Choudhary, Y. A. Manjunatha, Priyadarshini M. M.

1. Associate Professor, Department of Pathology, Dr. B. R Ambedkar Medical College, Bangalore.

2. Professor \& HOD, Department of Pathology, Dr. B. R Ambedkar Medical College, Bangalore.

3. Post Graduate, Department of Pathology, Dr. B. R Ambedkar Medical College, Bangalore.

\section{CORRESPONDING AUTHOR}

Dr Shaista Choudhary, No60, 10 th A Cross, West of Chord road, $2^{\text {nd }}$ stage, Bangalore-80, E-mail: drshaista5@rediffmail.com, Ph: 00919901474389.

ABSTRACT: Sarcoidosis is an uncommon disease in India and Cardiac Sarcoidosis is a known but uncommon manifestation of Sarcoidosis. The clinical features and presentations of cardiac sarcoidosis are protean and vary from benign ectopic to life threatening ventricular tachycardia. The outcome of most of the cases that are not diagnosed in time is sudden death. [1]

KEY WORDS: Cardiac sarcoidosis, interventricular septum, sudden death.

INTRODUCTION: Cardiac sarcoidosis occurs with more frequency in young adults[2]Bernstein et al were the first to recognise this entity. Gozo and his associates were the first to describe its clinical and pathologic features. Hagemann and Wurms estimated that the heart was affected in about $5 \%$ of patients with chronic sarcoidosis. ${ }^{[3]}$ But the actual incidence of cardiac sarcoidosis is much higher than the number of cases reported. This could be due to the lack of awareness of the disease with increased chances of misdiagnosis clinically. The incidence of sarcoidosis in developing countries like India has been underestimated, due to overwhelming incidence of tuberculosis which mimics this condition closely. Most of the affected patients are young adults and survival is limited to 2-3 years after cardiac involvement. The diagnosis based on clinical features is really difficult because of its varied presentation, ranging from benign arrhythmias to fatal ventricular fibrillations.[3]Therefore one has to be very careful while dealing with cases suspected to have any cardiac symptom .The same holds good even for cases with abnormal cardiac or pulmonary investigations.

CASE REPORT: We describe a case of myocardial involvement by sarcoidosis that lead to sudden death of a 38 years old man. He was found dead and autopsy was conducted to know the cause of death. Viscera were sent for histopathological examination. Grossly there was hypertrophy of heart (figure1).Cut surface of heart showed grey white patchy areas over the interventricular septum and extending towards right and left ventricular wall (figure2).Multiple sections were processed from the affected area. Microscopy revealed the presence of non caseating granulomas with numerous giant cells in the background (figure3).Gross appearance of lungs was unremarkable. The microscopy showed non caseating granulomas with giant cells in the lungs too (figure4). The autopsy report was finalised as cardiac sarcoidosis to be the cause of sudden death after ruling out other causes of granulomatous lesions affecting the heart. Since it was a post-mortem case, investigation reports were not available.

Journal of Evolution of Medical and Dental Sciences/Volume1/ Issue4/October - 2012 Page 504 
DISCUSSION: Cardiac sarcoidosis is a potentially fatal condition and not frequently diagnosed during clinical examination. ${ }^{[4]}$ It is an incidental finding in autopsy cases. In necropsy studies of sarcoidosis it was found that the proportion of cases with cardiac involvement by granulomas was higher than the proportion of cases with cardiac sarcoidosis during life. There are various other causes for granulomas in the heart such as giant cell myocarditis, idiopathic granulomatous myocarditis, tuberculous myocarditis, fungal myocarditis, Whipple disease, S.L.E, rheumatoid nodule and Wegener's granulomatosis.

Idiopathic granulomatous myocarditis and giant cell myocarditis stand as two most important differential diagnosis for cardiac sarcoidosis. In case of idiopathic granulomatous myocarditis there are non caseating granulomas in the heart without granulomatous involvement of the other organs. In giant cell myocarditis on the other hand is characterized by an infiltrate of eosinophils, lymphocytes, macrophages and giant cells associated with myocyte necrosis. The macrophage does not aggregate to form granulomas. This can be appreciated in routine Hematoxylin-Eosin (H \& E) stained sections. Infective granulomatous myocarditis (tuberculous myocarditis) is quite rare and granulomas are of caseating type and have larger lymphocytes as compared to granulomas of sarcoidosis. Fungal infection of the heart can also give rise to granulomas but the fungal elements can be identified under routine stain because of the presence of plenty of inflammatory cells predominantly eosinophils and plasma cells.

In case of Cardiac Sarcoidosis there are non caseating granulomas in the cardiac tissue along with same features in other organs. Thus, the other causes of granulomatous inflammation of the heart can be ruled out. A.F.B (Acid fast Bacilli) stain on the representative tissue which was also negative. However the microscopic features on $\mathrm{H}$ and $\mathrm{E}$ itself were quite characteristic of the lesion ${ }^{[5]}$.Clinical evidence of myocardial involvement is present in approximately five percent of patients with sarcoidosis, although autopsy studies indicate that subclinical cardiac involvement is present in $20-30 \%$ of cases. R. Guleria et.al in their article have discussed the varied presentations of cardiac sarcoidosis.[1]The clinical features may vary between body ache, dyspnoea, palpitations, fatigue etc .It is advisable to start with preliminary investigations like chest X-ray, pulmonary function tests and ECG. Transbronchial lung biopsy can be done in patients with hilar lymphadenopathy. Cardiac biopsy can be performed in few cases which show e.g. any abnormality. Investigators have recommended that all patients of sarcoidosis must be investigated for cardiac involvement with halter and thallium scan.

Cardiac sarcoidosis is a disease of remission and relapse. Cardiologist and pulmonologist have to work up the case together in order to reach an early diagnosis. ${ }^{[1]}$ If the patient presents with cardiac and respiratory symptoms and pulmonary investigations point towards sarcoidosis of lung, then it is mandatory to have high suspicion towards cardiac sarcoidosis, provided the patient also has cardiac symptoms.

Cardiac biopsy is very specific but on rare occasions diagnosis can still be missed because of the patchy distribution of the disease. Increased numbers of cases of cardiac sarcoidosis are being incidentally reported at autopsy [6], so one has to be careful in diagnosing subclinical cases. This case is being presented here for its academic interest and also to emphasize on subclinical cases which go unnoticed because of lack of awareness. 


\section{ORIGINAL ARTICLE}

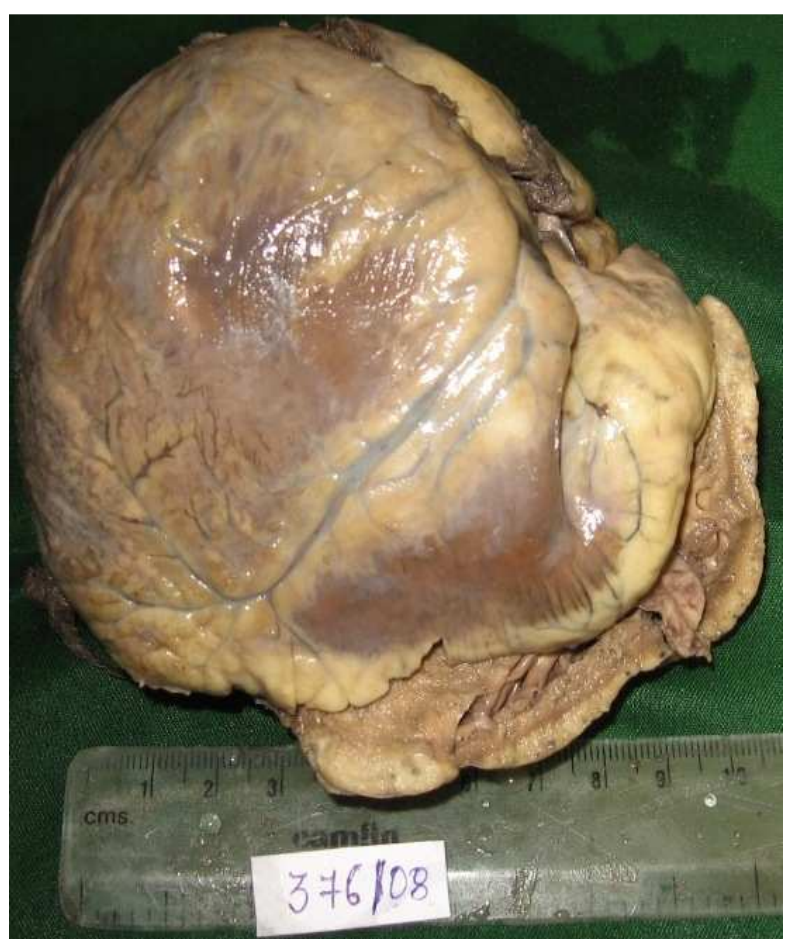

Figure-1: Gross appearance of autopsy specimen showing hypertrophy of heart.

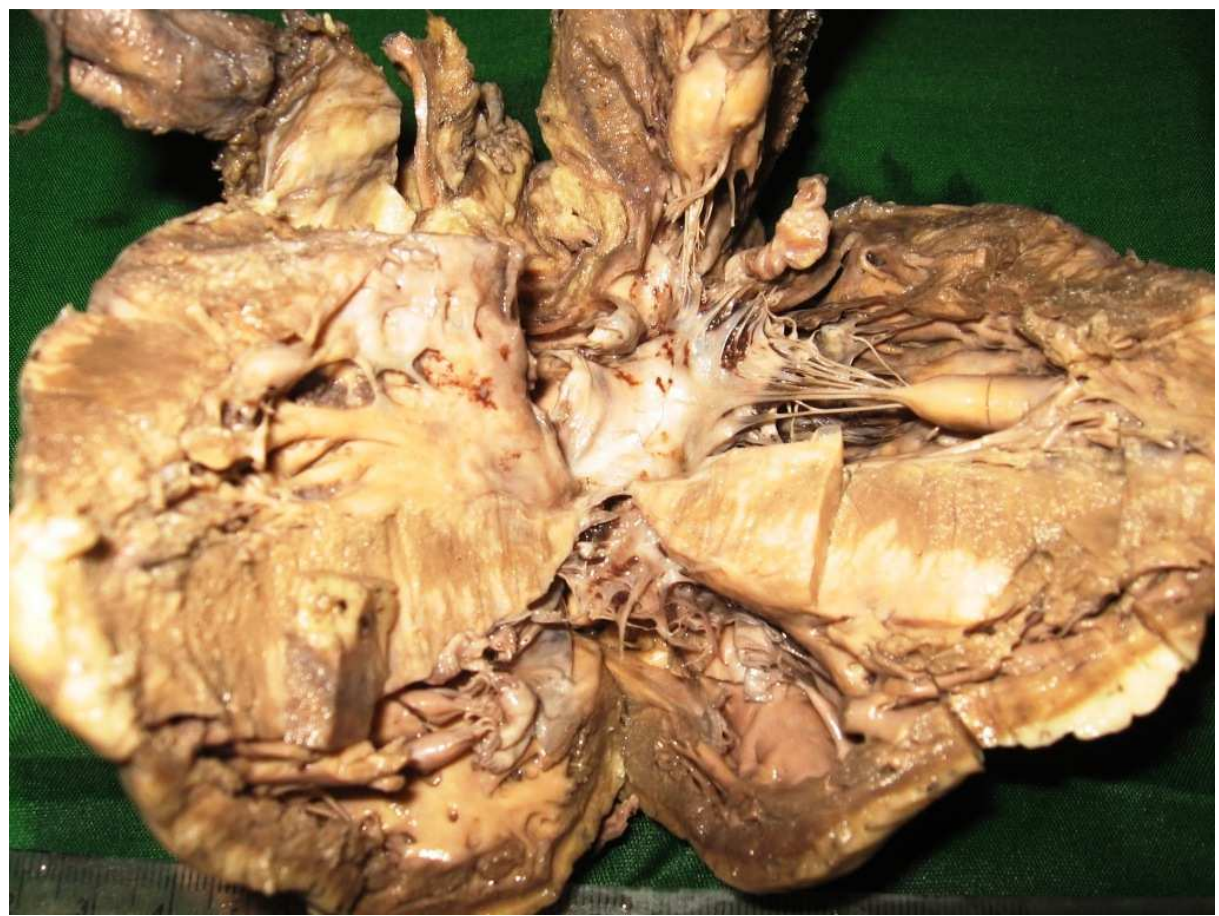

Figure-2: Cut surface show grey-white areas with thickening of interventricular septum. 


\section{ORIGINAL ARTICLE}

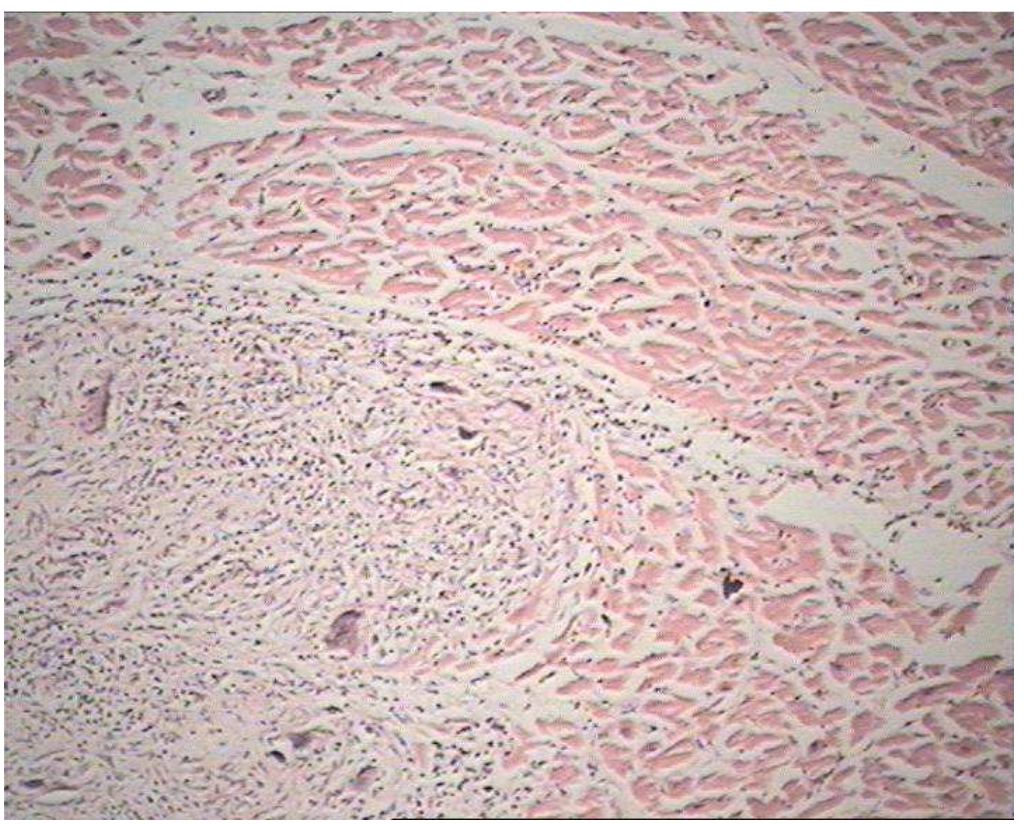

Figure-3: Show non-caseating granulomas in the cardiac muscle.

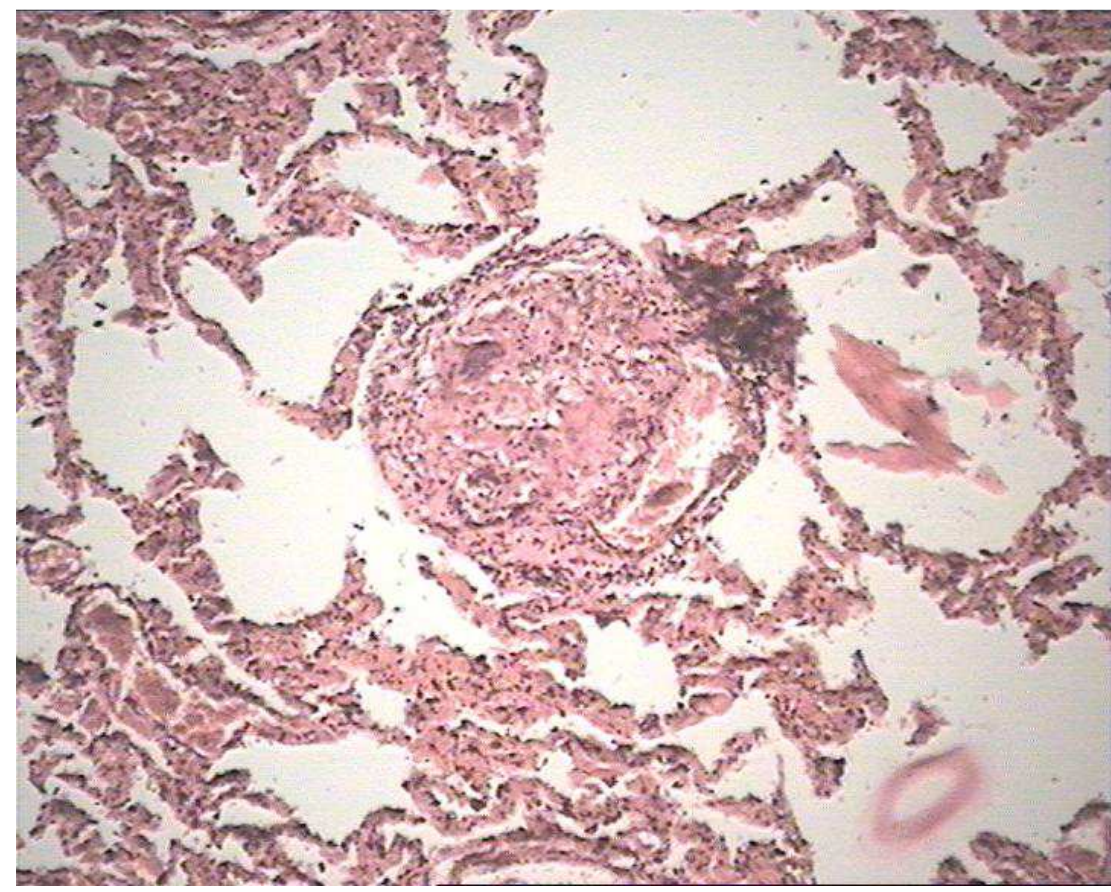

Figure-4: Show granuloma with giant cells in the lung parenchyma. 


\section{REFERENCES:}

1. Guleria R, Sharma R, Mohan A and Das C. Cardiac Sarcoidosis. Ind journal of Chest disease allied sci 2006; 48:133-37.

2. Wan Muhaizan WM, Swaminathan M, David M S. Cardiac Sarcoidosis-two cases with autopsy findings. Malays journal of pathol 2004; 26(1):59-63.

3. Sharma Om P. Diagnosis of cardiac sarcoidosis-An imperfect science: A hesitant art. CHEST 2003; 123(1) :18-19.

4. Kim Jessica S, Judson Mark A, Domino Robert, Gold Michael, Cooper Leslie T. Prystowsky Eric N et.al. Cardiac Sarcoidosis. American heart journal 2009; 157(1):9-21.

5. Lagna S M, Parvani A V , Nichols L C. Cardiac Sarcoidosis: a pathology focussed review. Arch Pathol Lab Med.2010; 134(7):1039-46.

6. Iwai Kazuro, Tachibana Jeruo, Takemura Tamiko, Matsui Yasuo, Kitalchi Masanori, Kawabata Yoshenori. Pathological studies on Sarcoidosis autopsyepidemiological features of 320 cases in Japan. Pathology international. ; 43(78):372-76. 